\title{
LASERS
}

\section{Topological effect}

Topological defects have been extensively studied in nematic liquid crystals and colloids. However, these structures do not support optical resonances because light confinement at naturally occurring topological defects is very weak due to the small size of liquid crystal molecules ( $2 \mathrm{~nm})$ and low refractive-index modulation.

Now, by combining the topological defect with a photonic defect cavity, Sebastian Knitter and colleagues from Yale University, NIST and University of Maryland in the USA have experimentally demonstrated the formation of strongly confined optical resonances with high quality factors (J. Opt. 18, 014005; 2016).

The team had previously looked into topological defects in liquid crystals, and learned how people created angular momentum by passing an optical beam through such passive samples with no gain. They also investigated lasing in random and ordered photonic structures and wondered whether they could use the topological defect structures to create novel lasing states by making active samples.

In their current work, they incorporated the topological defect structure into a regular photonic crystal defect cavity by making the unit cell anisotropic. Specifically, they considered a square lattice of elliptically shaped air holes in a dielectric membrane (pictured).

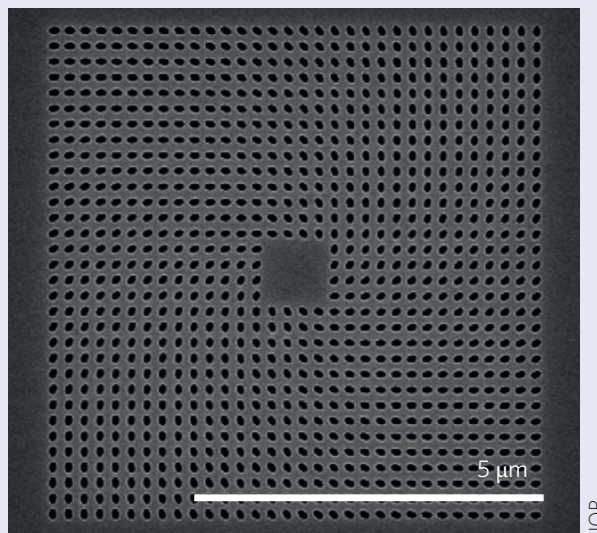

By manipulating the ellipse orientation, the energy flow inside the photonic crystal can be controlled, for example, to generate an optical vortex. The localized optical resonances in the topological defect structure can exhibit a tightly confined circulating flux pattern, in contrast to the outward energy flow of the regular photonic crystal defect state. By adding optical gain to such a structure, by means of embedding three uncoupled layers of InAs quantum dots, equally spaced by $25-\mathrm{nm}$ GaAs barriers, inside the $\mathrm{GaAs}$ layer, the team realized lasing in the vortex mode. Both numerical simulations and experimental tests were carried out.

A 190-nm-thick GaAs layer and a 1,000-nm-thick $\mathrm{Al}_{0.75} \mathrm{Ga}_{0.25}$ As layer were grown on a GaAs substrate by molecular beam epitaxy. The dimensions of the air holes as well as the lattice constant are on the order of the optical wavelength. The angular orientation of the elliptical holes varies spatially to produce a topological defect. They introduced a distinct orientational alignment of the ellipse, resembling that of rod-shaped molecules in nematic liquid crystals. This breaks the translational symmetry of the photonic crystal, representing a new type of defect. To enhance light confinement, $4 \times 4$ air holes were removed from the centre of the topological defect structure, creating localized states analogous to the photonic defect states. The team disclosed that, however, there is a trade-off between the vortex strength and the quality factor of the mode, which determines the lasing threshold.

"The general implication of this work is that the spatially inhomogeneous variation of the unit cell orientation can be used as [an] additional degree of freedom to control a lasing mode, enabling the manipulation of its field pattern and energy flow landscape. In terms of practical application, the optical vortex of the lasing mode may potentially be useful for onchip nanoparticle manipulation," said Hui Cao, the team leader, to Nature Photonics.

RACHEL WON 